\title{
Conscious Sedation in Plastic Surgery: Patient Safety and Cost Reduction Midazolam/Meperidine Conscious Sedation
} \author{
Abou Zeid ${ }^{3}$ \\ ${ }^{1}$ Resident, Department of Plastic and Reconstructive Surgery, Hotel Dieu de France Hospital, Lebanon \\ ${ }^{2}$ Assistant Professor, Department of Plastic and Reconstructive Surgery, Hotel Dieu de France Hospital, Lebanon \\ ${ }^{3}$ Attending in the Plastic and Reconstructive Surgery, Hotel Dieu de France Hospital, Lebanon \\ ${ }^{4}$ Professor, Department of plastic and reconstructive surgery, Hotel Dieu de France Hospital, Lebanon \\ ${ }^{5}$ Head of Department Plastic and Reconstructive Surgery, Hotel Dieu de France Hospital, Lebanon
}

Samer E Haber ${ }^{1}$, Marwan Nasr ${ }^{2}$, Fadi Sleilati ${ }^{3}$, Fady Ferran ${ }^{4}$, Nabil Hokayem ${ }^{5}$, Joseph El Khoury ${ }^{*}$ and Samer

Submission: March 26, 2018; Published: May 02, 2018

*Corresponding author: Joseph El Khoury, Department of Plastic and Reconstructive Surgery, Hotel Dieu de France Hospital, Alfred Naccache Blvd, Achrafieh, Beirut 1100 2160, Lebanon, Email: drjosephelkhoury@gmail.com

\begin{abstract}
Background: Conscious sedation is an anesthetic modality used in different procedures, among which are plastic surgeries. This study evaluated the efficacy and safety of the IV Midazolam - IM Meperidine conscious sedation protocol.

Methods: Adult patients undergoing elective cosmetic surgeries were enrolled. Patients were excluded if they had a history of cardiac or respiratory disease or in case of pregnancy. Patient anxiety, nausea, and pain were evaluated during and after surgery. Physician satisfaction and reported intraoperative level of sedation were also assessed. Operative time, midazolam dose, and the lowest hemoglobin saturation level in oxygen were noted. The primary outcome was postoperative recovery time while cost reduction and post operative complications were considered as secondary outcome measures.

Results: 173 patients were recruited. $61.8 \%$ under went rhinoplasty procedures and $13.8 \%$ under went blepharoplasty. Mean recovery time was 196 minutes. Age was the most significant predictor of recovery time. Older patients recovered faster $(p=0.019)$, were less anxious preoperatively $(\mathrm{p}=0.003)$ and had less postoperative pain $(\mathrm{p}=0.007)$ and nausea $(\mathrm{p}<0.0001)$. Higher dose of Midazolam were associated with more intraoperative anxiety $(\mathrm{p}=0.02)$ and an increased postoperative nausea $(\mathrm{p}=0.047)$ and emesis $(\mathrm{p}=0.032)$. We also can note that High $(7-14$ glasses/week) alcohol intake was associated with slower discharge $(\mathrm{p}=0.022)$.

Conclusions: The IM Meperidine-IV Midazolam conscious sedation protocol in plastic surgery operations in the one-day-surgery setting seems to be safe reproductible and cost effective.
\end{abstract}

\section{Introduction}

Conscious sedation or "Procedural Sedation and Analgesia (PSAA)" is the modality in which sedative agents are administered (with or without analgesics) to induce a minimally depressed level of consciousness sufficient to perform certain procedures. The strict definition requires that the patient be responsive to verbal command at all times and maintain airway patency and protective reflexes. It has been used across numerous specialties to perform invasive surgical, endoscopic, and intravascular procedures of limited durations; these include orthopedic, oral, plastic, neuroendovascular, cardiac, gastric, and radiological procedures [1-8]. The technique has been widely employed in plastic surgery thanks to the advantages it has over other anesthetic modalities, namely lower costs, faster recovery, better patient and surgeon satisfaction, and lower morbidity [9]. The surgical use of conscious sedation requires the concomitant administration of local anesthetic agents in order to achieve appropriate analgesia in the intraoperativeas well as the postoperative setting. Conscious sedation technique aims at attaining three main objectives when employed in plastic surgery: to reduce or eliminate the pain associated with injection of local anesthetic, to reduce or eliminate patient apprehension, and to reduce or eliminate recall of the operation [10]. This study aims to describe and evaluate the safety and efficacy of an IV Midazolam-IM Meperidine (Pethidine) conscious sedation protocol that has been in use for three decades over thousands of patients at our institution in patients under going elective cosmetic surgeries.

\section{Patients and Methods}

\section{Ethics statement}

Institutional Review Board approval was obtained. Patients aged eighteen years or older undergoing elective surgical procedures under conscious sedation provided their written informed consent to participate in the study. 


\section{Study design and patient selection}

This prospective study recruited patients at the Department of Plastic Surgery in major tertiary University Hospital in Beirut from November 2013 to December 2014. Patients aged eighteen years or older undergoing elective cosmetic surgical procedures, in which the Midazolam/Meperidine conscious sedation protocol is usually used, were approached and the study objectives were explained to each patient.. Patients were excluded if they had a history of cardiac or respiratory disease or in case of pregnancy.

\section{Surgical techniques and conscious sedation protocol}

Premedication was administered at the day care unit around thirty minutes before surgery as an intramuscular injection of Meperidine $75 \mathrm{mg}$, Haloperidol $2.5 \mathrm{mg}$, and Atropine $0.25 \mathrm{mg}$. Surgical procedures were conducted in a specially conceived operating room where the primary surgeon and his assistant - a resident - operate in the presence of one circulating nurse. Anesthesiologists were located nearby, readily available for intervention upon request.

In blepharoplasty procedures, oxybuprocaine eye drops (Cebesine $0.4 \%$ ) were used in the immediate preoperative period while in rhinoplasty procedures, a lidocaine-based gel was applied intranasally before in filtration by the local anesthetic solution (Lidocaine 1\% with 1/200,000 epinephrine).

After positioning and proper draping, a direct intravenous Midazolam dose ( 1 to $3 \mathrm{mg}$ ) was administered seconds before the infiltration by the local anesthetic solution. Sedation level could be readily verified through mild non-verbal stimulation by gently touching the patient's eyelashes and looking for a reflex response. Infiltration was then undergone, and in rhinoplasty, the nose was packed with gauze soaked in Lidocaine-based gel. Oxygen saturation level was monitored throughout the procedure and the levels of sedation and anxiety were constantly evaluated. If the patient became or remained conscious and/or anxious, the surgeon called for additional midazolam as needed. On the other hand, if the patient's respiratory rate and/or oxygen saturation decreased, the patient was verbally instructed to breathe deeply. In case the patient was not responsive to verbal stimulation, a brief jaw-thrust maneuver was applied, or, very rarely, a Guedel cannula could be inserted for airway patency or reverse medication could be administered.

The postoperative period consisted of a minimum of three hours of rest during which the patient wasn't allowed nothing per orem. After this recovery period, when the patient could autonomously walk and eat, he/she were considered ready for discharge.

\section{Patient and surgeon questionnaires}

Patient anxiety was evaluated before and after premedication (auto-evaluation and evaluation by the physician). Physician satisfaction was noted on a scale from zero to ten, as well as intraoperativelevel of sedation (Modified Observer's Assessment of Alertness/Sedation Scale) [11] at four different timepoints during surgery: 1- at infiltration of the local anesthetic, 2- fifteen minutes after the infiltration, 3- during lateral osteotomy (only in rhinoplasty procedures), and 4- during cast placement (only in rhinoplasty) (Table 1). Intraoperative nausea or emesis was also assessed. Operative time, midazolam dose, and the lowest hemoglobin saturation level in oxygen were noted. Postoperative anxiety, nausea/emesis, and pain were evaluated using a questionnaire employed in a study by Hasen et al. [12] The designated primary outcome was recovery time which corresponded to the time needed to regain orthostatic autonomy after the end of the operation. Discharge time - time from the end of the operation until discharge - depended less on the autonomy of the patient than on administrative and logistic issues, which meant that it was less related to the recovery from sedation than recovery time. Cost reduction, unintended admissions, pain, anxiety, nausea, emesis, and patient recall were considered the secondary outcome measures.

Table 1: Modified Observer's Assessment of Alertness/Sedation Scale.

\begin{tabular}{|c|c|}
\hline Responsiveness & Score \\
\hline Agitated & 6 \\
\hline $\begin{array}{c}\text { Responds readily to name spoken in normal tone } \\
\text { ("Alert") }\end{array}$ & 5 \\
\hline Lethargic response to name spoken in normal tone & 3 \\
\hline Responds only after name is called loudly and/or \\
repeatedly & 2 \\
\hline Responds only after mild prodding or shaking & 1 \\
\hline Does not respond to mild prodding or shaking & 0 \\
\hline Does not respond to deep stimulus & 3 \\
\hline
\end{tabular}

\section{Statistical analysis}

Statistical analyses were done using Stata. KolmogorovSmirnov test was undergone to test for the normality of the distribution of independent variables. Statistical relationships were studied between dependent variables and independent variables.

\section{Results}

A total of 173 patients were recruited of which 144 were females (83.2\%). Mean age was 35.2 years (95\% confidence interval [32.8; 37.6] years) (Table 2). 107 patients (61.8\%) underwent rhinoplasty procedures, 24 patients $(13.8 \%)$ underwent blepharoplasty procedures and 42 patients $(24.3 \%)$ under went other procedures (liposuction/fat grafting, otoplasty, and other procedures). Mean operative time was 59.5 minutes (95\% confidence interval (CI) 56.3-62.8 minutes). Mean recovery time was 196 minutes (95\% CI 181-212). None of the patients required intraoperative anesthesiologist intervention or an unintended admission. $90 \%$ of patients recalled some events from the operation while only $10 \%$ recalled an intraoperative bad experience. $10.5 \%$ of patients reported 
postoperative nausea. However, when asked to rate their nausea from 0 to 10 , only $15.4 \%$ of 155 patients reported a score of more than 5. Postoperative emesis was reported in $14 \%$ of patients. One patient presented to the emergency department 4 hours after discharge for extrapyramidal symptoms (trismus). She was successfully treated with a $50 \mathrm{mg}$ intramuscular dose of promethazine (Phenergan $囚)$. Other results are summarized in Tables 2-4.

Table 2: Descriptive statistical data.

\begin{tabular}{|c|c|c|}
\hline Variable & Mean & 95\% CI* \\
\hline Age (years) & 35.2 & $32.8-37.6$ \\
\hline $\begin{array}{l}\text { Anxiety before premedication } \\
\text { (scale from } 0 \text { to } 10 \text { ) }\end{array}$ & 4.7 & $4.2-5.2$ \\
\hline $\begin{array}{l}\text { Anxiety after premedication } \\
\text { (scale from } 0 \text { to } 10 \text { ) }\end{array}$ & 3.8 & $3.2-4.4$ \\
\hline Duration of operation (minutes) & 59.5 & $56.3-62.8$ \\
\hline Midazolam dose (mg) & 4 & 3.8- 4.3 \\
\hline Minimum 02 saturation (room air, \%) & 90.1 & 87.8-92.5 \\
\hline $\begin{array}{l}\text { Surgeon satisfaction } \\
\text { (scale from } 0 \text { to } 10 \text { ) }\end{array}$ & 9 & 8.4- 9.6 \\
\hline $\begin{array}{l}\text { Patient satisfaction } \\
\text { (scale from } 0 \text { to } 10 \text { ) }\end{array}$ & 8 & 7.6- 8.4 \\
\hline $\begin{array}{l}\text { Postoperative nausea score }>5 \\
\text { (scale from } 0 \text { to } 10)(n=155)\end{array}$ & $24(15.4 \%)$ & \\
\hline Postoperative emesis (n=155) & $22(14 \%)$ & \\
\hline $\begin{array}{l}\text { Postoperative pain score }>6 \\
\text { (scale from } 0 \text { to } 10)(n=159)\end{array}$ & $23(14.5 \%)$ & \\
\hline Recovery time (minutes) & 196 & $181-212$ \\
\hline Discharge time (minutes) & 325 & $0-750.7$ \\
\hline
\end{tabular}

${ }^{*} \mathrm{Cl}$ : Confidence Interval.

Table 3: Modified Observer's Assessment of Alertness/Sedation Scale.

\begin{tabular}{|c|c|c|}
\hline Variable & Mean & $\mathbf{9 5 \%} \mathbf{C I}^{*}$ \\
\hline $\begin{array}{c}\text { Responsiveness during infiltration of local } \\
\text { anesthetic }\end{array}$ & 2.9 & $2.7-3.2$ \\
\hline $\begin{array}{c}\text { Responsiveness during the procedure } \\
\text { Responsiveness during osteotomy in } \\
\text { rhinoplasty (n=107) }\end{array}$ & 2.6 & $2.4-2.8$ \\
\hline $\begin{array}{c}\text { Responsiveness during cast placement in } \\
\text { rhinoplasty (n=107) }\end{array}$ & 3.3 & $2.9-2.6$ \\
\hline
\end{tabular}

${ }^{*} \mathrm{Cl}$ : Confidence Interval.
Table 4: Patient recall of intraoperative events.

\begin{tabular}{|c|c|c|}
\hline Variable & Mean & 95\% CI* \\
\hline $\begin{array}{c}\text { Recall entering the operating room } \\
\text { (before IV midazolam) (n=157) }\end{array}$ & $146(93 \%)$ & \\
\hline $\begin{array}{c}\text { Recall events from the operation itself } \\
(\mathrm{n}=157)\end{array}$ & $90(67 \%)$ & \\
\hline $\begin{array}{c}\text { Recall peroperative bad experience } \\
(\mathrm{n}=158)\end{array}$ & $16(10 \%)$ & \\
\hline $\begin{array}{c}\text { Recall peroperative pain (n=157) } \\
\text { Recall peroperative nausea (n=155) }\end{array}$ & $22(14 \%)$ & \\
\hline Recall peroperative emesis (n=152) & $4(3 \%)$ & \\
\hline Recall peroperative anxiety (n=146) & $36(25 \%)$ & \\
\hline Peroperative anxiety score $(0-10)(n=151)$ & 3.8 & $3.2-4.3$ \\
\hline
\end{tabular}

${ }^{*} \mathrm{Cl}$ : Confidence Interval.

\section{Primary and secondary outcomes}

In univariate analyses, age was the most significant predictor of recovery time. Older patients recovered faster $(p=0.019)$, and were discharged faster $(p=0.009)$. They were less anxious preoperatively (before $(\mathrm{p}=0.003)$ and after premedication $(p=0.092)$, had less postoperative pain $(p=0.007)$, nausea $(\mathrm{p}<0.0001)$, and emesis $(\mathrm{p}=0.006)$, and showed deeper sedation at the end of the rhinoplasty operations during cast placement $(p=0.008)$. There was a trend towards better satisfaction with sedation in older patients $(\mathrm{p}=0.092)$.

Male patients were less anxious preoperatively both before $(p=0.0027)$ and after $(p=0.069)$ premedication, and intraoperatively (with significant patient recall of intraoperative anxiety). In rhinoplasty procedures, they were more sedated during osteotomy $(p=0.0004)$ and during cast placement $(p=0.035)$. They also had less vomiting episodes postoperatively $(\mathrm{p}=0.021)$. An average of $90.1 \%$ minimal oxygen saturation $(95 \%$ confidence interval, 87.8-92.5) was noticed in all patients.

Midazolam dose was increased when intraoperative patient anxiety was reported to be high by the surgeon $(\mathrm{p}=0.011)$, and it was higher in patients with less deep sedation at cast placement in rhinoplasty procedures $(\mathrm{p}=0.046)$. Patients who had received a higher dose of Midazolam reported more intraoperative anxiety $(p=0.02)$ and less recall of entering the operating room $(p=0.0024)$. Higher midazolam dose was associated with increased postoperative nausea $(p=0.047)$ and emesis $(p=0.032)$.

Analgesic intake in the past two weeks tended to be associated with recall of events from the operation (Chi-2 test; p=0.05). 29 of 55 patients who had taken analgesics did not recall events from the operation (53\%) versus 36 of 99 patients who had taken analgesics (36\%).

High (7-14 glasses/week) alcohol intake was associated with slower discharge $(p=0.022)$. In our establishment the fixed 
hospital cost In a conscious sedation protocol for blepharoplasty and rhinoplasty is around $700 \$$ where it is $1700 \$$ in a general anesthesia setting. This means a 59\% cost reduction.

\section{Discussion}

Conscious sedation is an anesthetic technique where the patient's consciousness level is diminished while maintaining response to verbal command. Airway protection reflexes, muscle contraction and thermal regulatory mechanisms are still present thus preventing aspiration, deep vein thrombosis (DVT), and hypothermia [3].

None of our patients required intraoperative anesthesiologist intervention or an unintended admission. Marcus et al. [1] reported 15 unintended admissions (out of 300 patients) in their study using the fentanyl/midazolam conscious sedation protocol, $73 \%$ of which was due to nausea and vomiting. Gart et al. [3] suggested that although the rate of unintended readmission was low in conscious sedation patients, high doses of preoperative diazepam decreased intraoperative midazolam and fentanyl use and reduced the incidence of postoperative nausea/vomiting. This strategy was found to decrease the rate on unintended admissions due to nausea and vomiting from around $1 \%$ to $0 \%$.

In our study, the mean recovery time was 196 minutes (95\% CI 181-212). This result is similar to that observed in a study comparing bolus to continuous midazolam administration (A). The recovery time for procedures of 120-180 minutes duration was 90-240 minutes. However, in other studies, intraoperative IV fentanyl rather than meperidine yielded shorter recovery times (63 minutes) but requiring an anesthesiologist [6].

$90 \%$ of patients recalled some events from the operation while only $10 \%$ recalled an intraoperative bad experience. $10.5 \%$ of patients in our study reported postoperative nausea and $14 \%$ reported postoperative emesis. In a study comparing conscious to deep sedation, Hasen et al. [12] reported a $17 \%$ rate of recall of unpleasant intraoperative events in the conscious sedation group (vs. only $3 \%$ in the deep sedation group). However, both groups had low recall of intraoperative pain, anxiety, and nausea, and the deep sedation group experienced significantly more nausea in the recovery room ( $p=0.002)$, at the time of discharge $(p=0.009)$, and the evening after the operation $(p=0.013)$. Amnesia of intraoperative events confers conscious sedation a similar patient experience to that of general anesthesia. Absence of inhalational anesthetics and the low dose of narcotics decrease postoperative nausea and vomiting (PONV) [14]. PONV is often referred to by patients as the most unpleasing issue in the entire operative experience. It is closely associated with propofol and with high intraoperative opioid use which favors conscious sedation over general anesthesia - where propofol and high opioid doses are employed - and propofol-based deep sedation $[3,13]$. PONV incidence in benzodiazepine/opioid-based conscious sedation is reportedly higher amongst women and in the 6-16 years age group (34-51\%), with decreasing incidence with age (14-40\%) [14]. Our results fall into these limits with $15.4 \%$ of patients reporting a nausea score of 6 or more on a scale from 0 to 10, with female sex predisposition, and decreased PONV with advancing age.

In their study on fentanyl/midazolam conscious sedation, Marcus et al. [1] reported a negative correlation between recovery time and advancing $(\mathrm{p}<0.001)$. Similar results were obtained in our study where older patients recovered faster $(p=0.019)$, and were discharged faster $(p=0.009)$.

While general anesthesia remains the corner stone for most surgeries, it has a 1 to 50,000 fatality rate mainly due to thromboembolic events [14]. It also presents a risk of malignant hyperthermia and a $30 \%$ rate of post-operative nausea and vomiting despite proper anti-emetic measures, not to mention increased costs, hospital admissions, preoperative evaluation time, post-operative recovery time, and complications from oro-tracheal intubation (sore throat, tooth injury, atelectasis) $[1,10,13,14]$. On the other hand, conscious sedation decreases the risk of thromboembolic events without DVT prophylaxis $[9,15]$, decreases hospital stay, unintended admissions, and costs, and eliminates the need for a tracheal tube $[1,10,15,16]$.

Many protocols have been described for conscious sedation in plastic surgery; benzodiazepines are the most commonly used while propofol, ketamine and barbiturates are fairly found in some regimens [17-26]. Agents with a short duration of activity/ half-life are preferred and they are used in small incremental doses while constantly monitoring patient's alertness, respiratory rate and room air oxygen saturation. Multiple agents - propofol, ketamine, and midazolam - possess this property. However, the advantages that midazolam has include amnestic properties and reversibility, with increased patient satisfaction and safety, respectively. Propofol and Ketamine lack reversibility and their use by non-anesthesiologists remains controversial [15]. Midazolam is a short-acting benzodiazepine that provides sedation, anxiolysis, and antegrade amnesia and these effects are potentiated by opioid agents. The increased hypnotic effect of midazolam with age and in females is well documented in the literature $[4,5,27]$ and is clearly reflected in the results of this study. It should be noted that the need for sedation is not uniform throughout the procedure. An initial loading dose is required prior to the infiltration of the local anesthetic [10]. Once pain suppression is established, the depth of sedation needed becomes less important. The patient is hence allowed to gradually regain consciousness during the operation. Another bolus dose is usually added towards the end of rhinoplasty procedures prior to the lateral osteotomy to alleviate anxiety secondary to the sounds and vibrations generated by percussion. This explains why only $10 \%$ of our patients recalled peroperative bad experience while $67 \%$ recalled events from the operation. As for side effects, Midazolam-induced respiratory depression is primarily manifested by a decrease in respiratory rate while decreased oxygen saturation only comes next. Therefore, 
monitoring of the patient's oxygen saturation at room air but also of the respiratory rate must take place concurrently [28]. Our technique proved to be safe with an average of $90.1 \%$ minimal oxygen saturation (95\% confidence interval, 87.8-92.5).

More we can notice in Figure 1 that the septoplasty added to rhinoplasty increases the risk of post-operative nausea by 2.5 folds (Figure 1). We can also add that rhinoplasty in conscious sedation setting or general anesthesia if not associated with septoplasty present with similar post operative emesis and nausea. Finally a key factor to consider is a reduction of $60 \%$ of the patient hospital cost with no increase in complications and a earlier recovery than general anesthesia.

\section{Post operative emisis}

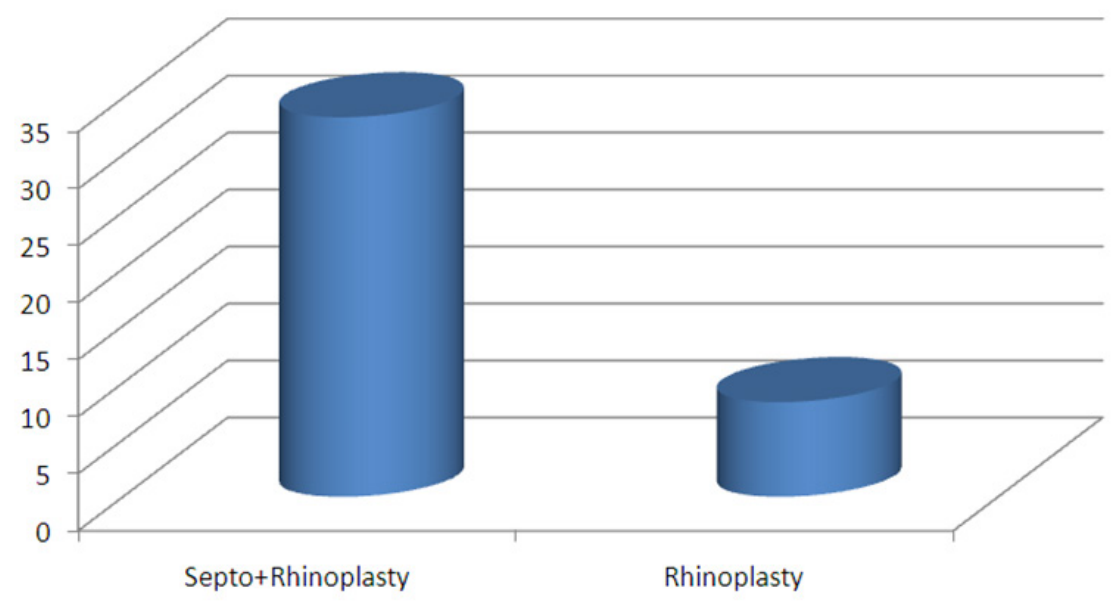

Figure 1: Comparison between the percentages of post-operative emesis between septoplasty associated to rhinoplasty and rhinoplasty alone.

\section{Conclusion}

This study provides further evidence on the safety and effectiveness of the IM Meperidine - IV Midazolam conscious sedation protocol in plastic surgery operations in the one-daysurgery setting. It has been used at our institution for more than twenty years over thousands of patients with no major complications occurring. Further studies are needed to compare the fentanyl/midazolam regimen with this meperidine/ midazolam protocol with or without dexmedetomidine in terms of safety, efficacy, and cost-effectiveness.

\section{References}

1. Marcus JR, Tyrone JW, Few JW, Fine NA, Mustoe TA (1999) Optimization of conscious sedation in plastic surgery. Plast Reconstr Surg 104(5): 1338-1345.

2. Hassan AE, Akbar U, Chaudhry SA, Tekle WG, Tummala RP, et al. (2013) Rate and prognosis of patients under conscious sedation requiring emergent intubation during neuroendovascular procedures. AJNR Am J Neuroradiol 34(7): 1375-1379.

3. Gart MS, Ko JH, Heyer KS, Mustoe TA (2013) Breast implant procedures under conscious sedation: a 6-year experience in 461 consecutive patients. Plast Reconstr Surg 131(5): 1169-1178.

4. Platten HP, Schweizer E, Dilger K, Mikus G, Klotz U (1998) Pharmacokinetics and the pharmacodynamic action of midazolam in young and elderly patients undergoing tooth extraction. Clin Pharmacol Ther 63(5): 552-560.

5. Czwornog J, Austin GL (2013) Body mass index, age, and gender affect prep quality, sedation use, and procedure time during screening colonoscopy. Dig Dis Sci 58(11): 3127-3133.
6. Hayee B, Rowbotham D, Saxena V, McNair A (2009) Fentanyl vs. pethidine as sedation for gastrointestinal endoscopy. Aliment Pharmacol Ther 30(3): 312-313.

7. Wagner HJ, Nowacki J, Klose KJ (1996) Propofol versus midazolam for sedation during percutaneous transluminal angioplasty. J Vasc Interv Radiol JVIR 7(5): 673-680.

8. Lee BS, Ryu J, Lee SH, Lee MG, Jang SE, et al. (2014) Midazolam with meperidine and dexmedetomidine vs. midazolam with meperidine for sedation during ERCP: prospective, randomized, double-blinded trial. Endoscopy 46(4): 291-298.

9. Reinisch JF, Bresnick SD, Walker JW, Rosso RF (2001) Deep venous thrombosis and pulmonary embolus after face lift: a study of incidence and prophylaxis. Plast Reconstr Surg 107(6): 1570-1575.

10. Baker TJ, Gordon HL (1988) Midazolam (Versed) in ambulatory surgery. Plast Reconstr Surg 82(2): 244-246.

11. Chernik DA, Gillings D, Laine H, Hendler J, Silver JM, et al. (1990) Validity and reliability of the Observer's Assessment of Alertness/Sedation Scale: study with intravenous midazolam. J Clin Psychopharmacol 10(4): 244-251.

12. Byun MY, Fine NA, Lee JY, Mustoe TA (1999) The clinical outcome of abdominoplasty performed under conscious sedation: increased use of fentanyl correlated with longer stay in outpatient unit. Plast Reconstr Surg 103(4): 1260-1266.

13. Hasen KV, Samartzis D, Casas LA, Mustoe TA (2003) An outcome study comparing intravenous sedation with midazolam/fentanyl (conscious sedation) versus propofol infusion (deep sedation) for aesthetic surgery. Plast Reconstr Surg 112(6): 1683-1689.

14. Keyes GR, Singer R, Iverson RE, McGuire M, Yates J, et al. (2008) Mortality in outpatient surgery. Plast Reconstr Surg 122(1): 245-250. 
15. Mustoe TA, Buck DW, Lalonde DH (2010) The safe management of anesthesia, sedation, and pain in plastic surgery. Plast Reconstr Surg 126(4): 165e-176e.

16. Hausman LM (2003) Advances in office-based anesthesia. Curr Opin Anaesthesiol 16(4): 421-427.

17. White PF, Vasconez LO, Mathes SA, Way WL, Wender LA (1988) Comparison of midazolam and diazepam for sedation during plastic surgery. Plast Reconstr Surg 81(5): 703-712.

18. Faymonville ME, Fissette J, Mambourg PH, Roediger L, Joris J, et al. (1995) Hypnosis as adjunct therapy in conscious sedation for plastic surgery. Reg Anesth 20(2): 145-151.

19. Michelson LN, Lindenthal JJ, Peck GC, Agresti CJ, Ship AG, et al. (1987) Diazepam and fentanyl as adjuncts to local anesthesia. Aesthetic Plast Surg 11(1): 207-211.

20. Chye EP, Young IG, Osborne GA, Rudkin GE (1993) Outcomes after same-day oral surgery: a review of 1,180 cases at a major teaching hospital. J Oral Maxillofac Surg 51(8): 846-849.

21. Moscona RA, Ramon I, Ben-David B, Isserles S (1995) A comparison of sedation techniques for outpatient rhinoplasty: midazolam versus midazolam plus ketamine. Plast Reconstr Surg 96(5): 1066-1074.

This work is licensed under Creative Commons Attribution 4.0 Licens

DOI: 10.19080/JHNSS.2018.02.555598
22. Friedberg BL (1999) Propofol-ketamine technique: dissociative anesthesia for office surgery (a 5-year review of 1264 cases). Aesthetic Plast Surg 23(1): 70-75.

23. Wu W, Chen Q, Zhang LC, Chen WH (2014) Dexmedetomidine versus midazolam for sedation in upper gastrointestinal endoscopy. J Int Med Res 42(2): 516-522.

24. Sethi P, Mohammed S, Bhatia PK, Gupta N (2014) Dexmedetomidine versus midazolam for conscious sedation in endoscopic retrograde cholangiopancreatography: An open-label randomised controlled trial. Indian J Anaesth 58(1):18-24.

25. Shen S, Zheng J, Zhang J, Wang W, Jin T, et al. (2013) Comparison of dexmedetomidine and propofol for conscious sedation in awake craniotomy: a prospective, double-blind, randomized, and controlled clinical trial. Ann Pharmacother 47(11): 1391-1399.

26. Fan TW, Ti LK, Islam I (2013) Comparison of dexmedetomidine and midazolam for conscious sedation in dental surgery monitored by bispectral index. Br J Oral Maxillofac Surg 51(5): 428-433.

27. Klotz U (1998) Effect of age on pharmacokinetics and pharmacodynamics in man. Int J Clin Pharmacol Ther 36(11): 581-585.

28. Pratt JM (1985) Analgesics and sedation in plastic surgery. Clin Plast Surg 12(1): 73-81.

\section{Your next submission with Juniper Publishers will reach you the below assets}

- Quality Editorial service

- Swift Peer Review

- Reprints availability

- E-prints Service

- Manuscript Podcast for convenient understanding

- Global attainment for your research

- Manuscript accessibility in different formats

( Pdf, E-pub, Full Text, Audio)

- Unceasing customer service

Track the below URL for one-step submission https://juniperpublishers.com/online-submission.php 